\title{
Die Grenzen des chinesischen Wirtschaftswachstums
}

Die Volksrepublik China (VRC) ist in der Weltwirtschaftsgeschichte ein außergewöhnlicher Fall. Nie zuvor hat ein Flächenstaat über dreieinhalb Jahrzehnte hinweg ein jährliches Wirtschaftswachstum von durchschnittlich rund zehn Prozent erreicht. Im letzten Jahrzehnt wurde China zum Kraftzentrum der Weltwirtschaft, während die etablierten OECD-Ökonomien weiter an Dynamik verloren und teils schwere Rückschläge erlitten. Darüber hinaus läuft Chinas Aufstieg unter der Herrschaft einer Kommunistischen Partei ab, die nach allen historischen Erfahrungen den denkbar ungünstigsten Kontext für wirtschaftspolitische Agilität, technologische Innovationskraft und globale Konkurrenzfähigkeit bieten müsste.

Trotz - oder gerade wegen - dieses beispiellosen Aufschwungs, der die VRC zur größten Handelsnation und zweitgrößten Volkswirtschaft der Welt werden ließ, sind Stimmen nie verstummt, die dem Land ein baldiges Ende des schnellen Wachstums vorhersagen. Seit den Jahren 2010 bis 2012, in denen in zehn aufeinanderfolgenden Quartalen das Wachstum des chinesischen Bruttoinlandsproduktes (BIP) in kleinen Schritten zurückging (bevor es sich seit Ende 2012 wieder beschleunigte), ist die Diskussion über Grenzen des chinesischen Wachstums neu entflammt.

In diesem Beitrag wird nicht eingegangen auf die mittels ökonometrischer Modelle - oftmals auf der Basis von realitätsfernen linearen Annahmen und immer weniger glaubwürdigen Gleichgewichtsmodellen (,growth accounting“) - geführte Kontroverse um die Frage, wie lange China mit welchen Wachstumsraten in $\mathrm{Zu}-$ kunft rechnen könne. Unter dem Stichwort „Grenzen des Wachstums“ sollen im Folgenden zentrale Risiken und Herausforderungen für die Zukunft des chinesischen Wirtschaftswachstums skizziert werden, wie sie die westliche Chinadebatte dominieren. Kernelemente dieser Debatte werden einer kritischen Prüfung unterzogen. Zugleich werden Thesen jenseits des ökonomischen Mainstreams zur Zukunftsfähigkeit der politischen Ökonomie Chinas begründet und zur Diskussion gestellt.

Die Diskussion um die ökologischen Kosten und Grenzen des chinesischen Wachstums wird nur gestreift, da es auf diesem Feld keine substanzielle Kontroverse gibt: Gravierende Umweltzerstörungen sind unbestreitbar. Die politischen und technologischen Handlungsnotwendigkeiten werden von der chinesischen Re- 
gierung anerkannt und in ambitionierten Umwelt- und Technologieprogrammen aufgegriffen. Inwieweit diese meist noch jungen Programme auf mittlere und lange Frist wirkungsvoll sind und inwieweit und auf welche Weise Umweltzerstörungen das chinesische Wachstum abbremsen werden, ist eine offene Frage, die in diesem Aufsatz angesichts der Vielzahl diesbezüglicher Erörterungen nicht behandelt werden muss.

\section{Das liberale Paradigma und die Grenzen des chinesischen Wachstumsmodells}

Die Diskussion um die Nachhaltigkeit des chinesischen Wirtschaftswachstums stützt sich auf eine typische Bandbreite sozialwissenschaftlicher Indikatoren: strukturelle und zyklische Einflussfaktoren, exogen und endogen induzierte Krisenpotenziale, realwirtschaftliche und monetäre Variablen, makroökonomische und mikroökonomische Risiken, ökologische Zerstörungen und Ressourcenengpässe sowie gesellschaftliche und politische Destabilisierungsanzeichen. Im Kern dreht sich die Diskussion um Defizite des bisherigen chinesischen Wachstumsmodells, die auch in China selbst weithin moniert werden und die mehr oder weniger explizit historische Erfahrungen von fortgeschrittenen OECD-Ökonomien und die daraus abgeleiteten wissenschaftlichen Referenzmodelle zum Maßstab nehmen.

Die Analyse der Schattenseiten des chinesischen Aufstiegs beschränkt sich in der Regel nicht darauf, die offensichtlichen oder vermeintlichen Risiken der Entwicklung zu benennen. Vielmehr suggerieren viele Wissenschaftler und Journalisten, dass sich eindeutige kausale Muster erkennen ließen und dass die konstatierten Missstände durch gezielte politische Maßnahmen dauerhaft zu beseitigen seien. Regelmäßig werden äußerst umfassende Vorschläge zur Restrukturierung der gesamten politischen Ökonomie formuliert, um die Zukunftsfähigkeit von Wirtschaft, Gesellschaft und Politik Chinas sicherzustellen. So betonen etwa die auf ein umfassendes „Rebalancing“ der chinesischen Wirtschaft gerichteten Empfehlungen ${ }^{1}$ typischerweise die folgenden Missstände und Reformerfordernisse:

- Das bisherige chinesische Wachstumsmodell beruhe einseitig auf Investitionen und Exportförderung und habe dadurch gravierende strukturelle Verzerrungen hervorgerufen. Die bisherigen Exporterfolge seien durch eine unterbewertete Währung ermöglicht worden und nicht länger aufrechtzuerhalten. Die Wettbewerbsfähigkeit gehe durch steigende Löhne und die Aufwertung des Chinesischen Yuan zurück und treffe zudem auf eine nachlassende Nachfrage aus Europa und den USA.

1 Repräsentativ und einflussreich sind zum Beispiel Lardy 2012, Lardy/Borst 2013 oder Pettis 2013. 
- Durch künstlich niedrig gehaltene Zinssätze („Finanzrepression“) der Staatsbanken würden in großem Umfang Kapitalströme von Sparern (insbesondere privaten Haushalten) zu privilegierten staatlichen Unternehmen (Geschäftsbanken und Industriekonzernen) gelenkt. "Finanzrepression" fördere die Aufrechterhaltung ineffizienter Unternehmens- und Marktstrukturen, Verfolgung unprofitabler Projekte und Entstehung von Investitionsblasen im städtischen Immobiliensektor. Durch eine politischen Kriterien folgende Kreditvergabepraxis habe sich ein riesiger Schuldenberg bei den lokalen Regierungen aufgetürmt. Die Gewinne der Staatsunternehmen würden künstlich aufgebläht. Private Unternehmen und Kleinanleger müssten in den Schattenbankensektor ausweichen. Der Dienstleistungssektor bliebe im internationalen Vergleich unterentwickelt.

Der Kern der Argumentation ist stets ähnlich: Je länger die Ungleichgewichte und Verzerrungen aufrechterhalten würden, desto kostspieliger und riskanter werde die anschließende Korrektur ausfallen. Als Ausweg bliebe nur ein neues Wachstumsmodell, das auf einheimische Nachfrage und Innovationen setzen müsse. Im Einzelnen wird empfohlen: der Rückzug des Staates aus Kernbereichen der Wirtschaft (Öffnung der Kapitalbilanz, Liberalisierung des Finanzsystems und des Wechselkurses, Beendigung der staatlichen Energiepreisfestsetzung), ein Ausbau der sozialen Sicherungssysteme und die Förderung unternehmensbasierter, außerhalb des staatlichen Zugriffs organisierter Forschung und Entwicklung.

Die Bedeutung einzelner der aufgeführten Indikatoren ist selbst unter neoklassisch orientierten Ökonomen durchaus umstritten. So wird die These des chinesischen „Overinvestments“ mit dem Argument zurückgewiesen, dass der existierende Kapitalstock Chinas im Vergleich zu westlichen Ökonomien noch sehr begrenzt sei und deshalb ausreichend Produktivitätsfortschritte in der Zukunft zu erwarten seien.

Auch die Konsequenzen der demografischen Wende (schnelle Alterung der Bevölkerung und sinkende Zahl nachwachsender Erwerbstätiger aufgrund der EinKind-Politik) und die daraus resultierende dämpfende Wirkung auf das zukünftige Wachstum könne man durchaus relativieren. Denn die noch ausstehende Urbanisierung Chinas (gerade einmal etwas mehr als die Hälfte der Bevölkerung lebt in Städten) dürfte noch auf Jahre hinaus den produktiveren städtischen Sektoren relativ billige Arbeitskräfte zuführen. Letztlich habe die chinesische Regierung durch ein bislang geringes Haushaltsdefizit, die hohe einheimische Sparrate und die hohen Devisenreserven ausreichend Finanzkraft, um den Übergang des Wachstumsmodells zu bewältigen.

Wir wollen im zweiten Teil dieses Beitrages bewusst über diese konventionelle Diskussion hinausgehen, indem wir einige grundsätzliche Einwände bezüglich der „Rebalancing“-Debatte formulieren. 


\section{Ein kontextspezifischer Blick auf politische Risiken und Krisen}

Zunächst sind einige epistemologische Bemerkungen angebracht. Die Zusammensetzung und die Interessen der Beobachter, die Chinas wirtschaftliche Entwicklung in der Öffentlichkeit vermitteln und beurteilen, haben sich fundamental verändert. So werden traditionelle akademische Analysen oder Forschungsberichte internationaler Organisationen (die aufgrund zeitaufwändiger Reviewverfahren oft mit erheblicher Verzögerung erscheinen) immer stärker in den Hintergrund gedrängt durch tagesaktuelle Beiträge von Research-Abteilungen internationaler Banken, Ad-hoc-Interviewstatements von medienerprobten Experten oder meinungsfreudige Weblogs mit einer großen Zahl von Followern.

Während Spezialinformationen über Chinas Wirtschaft noch vor wenigen Jahren nur in kleinsten Zirkeln Beachtung fanden, ergießt sich nun beinahe täglich ein breiter Informations- und Meinungsstrom zu den Rückwirkungen von Veränderungen im chinesischen Einkaufsmanager- oder Konsumentenpreis-Index, in städtischen Immobilienpreisen, Lagerbeständen von Schlüsselrohstoffen oder SWAPWechselkursen der chinesischen Währung. Nachrichten zur makroökonomischen Lage der VRC werden oft nicht mehr im Lichte von Fundamentaldaten und längerfristigen Trends beurteilt, sondern nur noch daran gemessen, inwieweit sie dem aktuellen „Market Sentiment“ entsprechen. Wie ein virales Lauffeuer verbreiten sich regelmäßig Internet-Berichte zu - nur selten auf den ersten Blick durchschaubaren oder überprüfbaren - Missständen in chinesischen Unternehmen oder Betrugsfällen im Zusammenhang mit Börsengängen chinesischer Firmen, die dann wieder von transnationalen Kapitalmarktakteuren (insbesondere durch Shortseller) für die eigenen Investitions- und Spekulationsszwecke genutzt werden können. Solche „Hidden Agendas“ werden begünstigt durch Informationsrepressionen aufseiten des chinesischen Staates und mangelnde Glaubwürdigkeit offizieller chinesischer Medien.

Ebenfalls problematisch sind Erfahrungsberichte von „Augenzeugen“, die beispielsweise „Geisterstädte“ in der Inneren Mongolei (in Form massenhaft leerstehender Büro- und Wohngebäude) oder „Geisterflotten“ in chinesischen Häfen (ungenutzt vor Anker liegende Containerschiffe) beschreiben, ohne die Generalisierbarkeit und zeitliche Gebundenheit solcher Phänomene zu thematisieren. Wenig aussagekräftige Momentaufnahmen dienen regelmäßig als Basis für generalisierende Aussagen über das chinesische "System" oder "Modell". Und aus isolierten Beobachtungen und Informationen werden systemische Reformerfordernisse abgeleitet. 
Ähnliches gilt für oft versteckt transportierte Prämissen oder Voreingenommenheiten in wissenschaftlichen Analysen. So tritt in vielen Beiträgen westlicher Forscher im Rahmen der „Rebalancing"-Debatte ein mehr oder minder offen formuliertes neoklassisches Paradigma aus Marktentfesselung, Wettbewerbsförderung, Deregulierung und Privatisierung zutage. Es handelt sich hierbei um ein kognitivparadigmatisches Repertoire, das im Kontext der gegenwärtigen Finanz- und Wirtschaftskrise vielfach diskreditiert ist und das die Realität umfassender Staatsinterventionen in westlichen Ökonomien nach 2007 ausblendet.

Die Generalisierungen des neoklassischen Standardmodells erscheinen mit Blick auf Chinas besondere Entwicklungserfahrungen kontextfremd und durch einen starken "Confirmation Bias" verzerrt: Erfahrungen und Modelle, die in westlichen Gesellschaften selbst zunehmend umstritten sind, leiten ungebrochen die Beurteilung Chinas an. Chinas politische Ökonomie wird a priori nicht zugestanden, exzeptionell oder innovativ zu sein. Vielmehr wird sie als abweichend und korrekturbedürftig im Lichte althergebrachter Modelle betrachtet, obwohl der technologisch-ökonomisch-kommunikative Kontext des 21. Jahrhunderts offensichtlich ganz neue Anforderungen an die Leistungs- und Anpassungsfähigkeit wirtschaftlicher und politischer Systeme stellt.

Besonders kritisch zu hinterfragen sind Studien (z. B. zur chinesischen Investitionsrate), die mittels Cross-Country Panel Estimations historische Regelmäßigkeiten und internationale Benchmarks identifizieren, anhand derer sodann chinesische „Abweichungen“ ermittelt und - gewöhnlich negative - Schlussfolgerungen für Chinas Zukunft abgeleitet werden. Oft werden in diesem Zusammenhang vermeintliche Muster im Vergleich zu früheren „Growth Stories“ oder historischen makroökonomischen Krisen mehr oder weniger ungeprüft auf China übertragen. Geradezu schablonenhaft-retrospektiv wird so beispielsweise immer wieder die Verschuldung der lokalen chinesischen Regierungen und die Gefahr einer Blase auf dem Immobilienmarkt als eine mögliche Wiederkehr der Subprime-Krise nach USamerikanischem Muster beschworen.

Solche Analogien gehen aber in einem zentralen Aspekt an der chinesischen Realität vorbei: Im Gegensatz zum Westen haben wir es in China in der Regel nicht mit privaten (im Ausland ansässigen) Gläubigern und Schuldnern zu tun, sondern mit staatlichen Akteuren auf beiden Seiten. Dadurch ist es staatlichen Stellen in China viel leichter möglich, unbedienbare Kredite zu verschleiern, zu überwälzen oder aus den Büchern zu tilgen. Chinas Banken- und Kapitalmärkte unterliegen also gänzlich anderen Verhaltensanreizen, Interaktions- und Rechenschaftsmechanismen als es OECD-Erfahrungen nahelegen. 
Fragwürdig ist auch die Praxis westlicher China-Korrespondenten, prominente chinesische Wissenschaftler oder Journalisten als Kronzeugen für die Notwendigkeit einer chinesischen Liberalisierungs- und Privatisierungsagenda ins Feld zu führen. Marktliberale Reformökonomen stellen nur einen Ausschnitt der sehr vielschichtigen und kontroversen innerchinesischen Debatte dar. Im Gegensatz zu den Reformökonomen, die dem westlichen "Confirmation Bias" entgegenkommen, werden die politisch wesentlich einflussreicheren Wirtschaftsplaner und Industriepolitiker der Regierungszentrale in der westlichen Chinabeobachtung weithin ignoriert, obwohl deren Positionen durch Publikationen und Konferenzbeiträge öffentlich zugänglich sind. ${ }^{2}$

Zur Erfassung der neuartigen ökonomischen, technologischen und politischen Bedingungen in der Welt des 21. Jahrhunderts geben die Schriften von Nassim Taleb grundsätzliche Hinweise. ${ }^{3}$ Taleb verdeutlicht die überwiegend nicht-linearen Wirkungsmechanismen in einem interdependenten globalen Wirtschafts- und Kommunikationssystem wie auch die herausragende Wichtigkeit von sozialwissenschaftlich nicht vorhersagbaren disruptiven Ereignissen für die Entwicklung menschlicher Gesellschaften. Zahlreiche seiner Überlegungen lassen sich produktiv auf die - sozialwissenschaftlich nicht vorhergesagten, mit althergebrachten Modellen nicht kompatiblen, aber global äußerst wirkungsmächtigen - Entwicklungserfahrungen Chinas anwenden.

Beispielsweise werden in westlichen Darstellungen die Wirtschaftskrisen der letzten 15 Jahre, die auch auf China einwirkten (Asienkrise, Dotcom-Krise, Subprime-Krise), in Verbindung mit hausgemachten chinesischen Krisenerscheinungen als Belastung für die Widerstands- und Erneuerungsfähigkeit (Resilienz) der politischen Ökonomie Chinas beschrieben. Taleb folgend könnten die von außen und innen auf das System einwirkenden „Stressoren“ jedoch als förderlich für die unerwartete Anpassungsfähigkeit oder „Antifragilität“ der politischen Ökonomie Chinas zu deuten sein: Die wiederkehrenden äußeren und inneren Herausforderungen zerstörten nicht die Kerninstitutionen des Systems, zwangen es aber immer wieder zu neuen Anpassungs- und Innovationsleistungen. Von exogenen Finanzkrisen bis hin zu endogenen sozialen Protesten: Die politische Ökonomie Chinas verdankt ihre administrative Agilität und makroökonomische Stabilität den Zwängen zu unkonventionellen, flexiblen Reaktionen auf begrenzte, nicht systembedrohliche Krisenherausforderungen.

3 Vergleiche Taleb 2007, ders. 2013. 
So hat sich in China seit den zyklischen Krisen der 1980er und 1990er Jahre ein unkonventionelles, aber effektives Instrumentarium zur Bearbeitung makroökonomischer Destabilisierungen herausgebildet, das unter dem Begriff der "Makrokontrolle" (hongguan tiaokong) die mittelfristige Entwicklungsplanung bzw. Industriepolitik sowie administrative Ad-hoc-Interventionen bis hin zu selektiven Preiskontrollen einschließt. Dieses stabilitätspolitische Instrumentarium geht weit über die begrenzten geld- und fiskalpolitischen Standardinstrumente hinaus, die westlichen Staaten zur Verfügung stehen. Chinas Regierung hat in der Stabilitätspolitik ein weitaus größeres Instrumentarium zur Verfügung als alle anderen gegenwärtigen Staaten und hat diese Steuerungswerkzeuge seit den 1990er Jahren beständig verfeinert.

Chinas politische Ökonomie hat sich über die bloße Widerstandsfähigkeit hinaus umfassend erneuert. Chinesische Entscheidungsträger haben westliche institutionelle Muster nicht ungeprüft und unverändert übernommen, sondern in einem über Jahrzehnte dauernden Einpassungsvorgang ausländische Erfahrungen selektiv für den chinesischen Kontext nutzbar gemacht, indem sie mit autochthonen Institutionen (etwa der Entwicklungsplanung und administrativen Interventionen) und zuvor etablierten Prozessmustern (inbesondere der Politikentwicklung "vom Punkt in die Fläche" durch Experimentalprogramme) amalgamiert wurden.

Die Anpassungsfähigkeit der hybriden, unkonventionellen Institutionen und Prozessmuster, die wir in Chinas politischer Ökonomie finden, hat sich bislang als unerwartet groß erwiesen - auch wenn diese Institutionen und Prozesse mit sozialwissenschaftlichen Standardmodellen und westlichen Erwartungen nicht kompatibel sind.

\section{Disruptive Krisenereignisse außerhalb strukturell-linearer Modelle}

Von den vorangegangenen Überlegungen ausgehend, identifizieren wir drei zentrale Risiken für die Zukunft des chinesischen Wirtschaftswachstums.

1. Wir sehen im Gegensatz zur Mehrheit westlicher Beobachter die Risiken der politischen Ökonomie Chinas weniger in strukturell-längerfristigen Verwerfungen als in ad hoc auftretenden Krisensituationen. Im Hinblick auf strukturelle Herausforderungen (etwa die Bereitstellung öffentlicher Güter und die Gestaltung des demografischen Übergangs) hat die chinesische Führung ausreichend Vorwarnzeit und mehr Handlungsmöglichkeiten als nahezu alle anderen gegenwärtigen Regierungen weltweit.

Viel gefährlicher sind Krisenszenarien, die die Regierung unvorbereitet treffen und deren Handlungsfähigkeit und Glaubwürdigkeit abrupt beschädigen können. 
Immer wieder kam es in solchen Fällen (vgl. SARS 2003 oder die fortgesetzten Lebensmittel- und Arzneimittelskandale) zu gravierendem Fehlverhalten der zuständigen Behörden: Anfängliche Krisensignale wurden negiert, unabhängige Nachrichten unterdrückt, entschlossenes Handeln wurde durch Delegation der Verantwortung an höhere Hierarchieebenen vermieden. In diesem Sinne entfalten auch die Umweltzerstörungen eine negative Wirkung auf Glaubwürdigkeit und Handlungsfähigkeit der nationalen Regierung, deren ambitionierte Umweltschutz- und Energieprogramme durch Ausweichverhalten lokaler Regierungen konterkariert wird. Jahrelang geduldete oder verschleierte Fehlentwicklungen können im Falle eines akuten, weithin sichtbaren und viral kommunizierten Ereignisses eine Systemkrise auslösen.

2. Im Falle von makroökonomischen Krisen hat die VRC seit den 1990er Jahren eine - im Vergleich zu allen anderen Schwellenländern - sehr effektive Stabilitätspolitik betrieben. Es ist allerdings fraglich, ob diese Durchschlagskraft in Zukunft noch im bisher gekannten Ausmaß gegeben sein wird, denn es gibt vielerlei Anzeichen dafür, dass die Autorität der Parteizentrale schleichend unterminiert wird. Informelle Patronage-, Tausch- und Familiennetzwerke höhlen die Parteihierarchie aus. Die Zersetzung der Parteihierarchie zeigt sich in grassierender Korruption bis in höchste Führungskreise hinein und im Transfer gewaltiger Kapitalvermögen durch Funktionärsfamilien ins Ausland.

Diese organisatorischen Zerfallstendenzen gehen einher mit wirtschaftlich und technologisch induzierten gesellschaftlichen Veränderungen. Durch die neuen Möglichkeiten zur interaktiven digitalen Kommunikation (Microblogging) ist in den städtischen Mittel- und Oberschichten ein Klima des Misstrauens und Zynismus entstanden, das im Falle eines wirtschaftlichen Einbruchs oder einer politischen Führungskrise nicht erwarten lässt, dass die Bevölkerung das bestehende Regierungssystem weiterhin akzeptiert oder passiv hinnimmt. Die Erwartungen aus der Gesellschaft an den chinesischen Staat sind dramatisch gewachsen. Sie beschränken sich nicht mehr nur auf wirtschaftliche Prosperität und politische Stabilität, sondern schließen heute auch ein umfassendes öffentliches Gesundheits- und Bildungswesen sowie die Sicherung guter Umweltbedingungen ein. Eine ganze Generation von Chinesen ist im Kontext eines rasanten Wachstums- und Modernisierungsprozesses aufgewachsen. Vor dem Hintergrund dieser Zukunftserwartungen könnten bereits kleinere Rückschläge eine abrupte Abkehr von der bestehenden Ordnung auslösen. 3. Besondere Gefahren ergeben sich für China durch den veränderten internationalen Kontext, wenn auch auf andere Weise als dies gewöhnlich in der westlichen Literatur thematisiert wird. Jenseits der viel diskutierten Großmachtpolitik besteht eine der virulentesten Herausforderungen in Chinas Außenbeziehungen darin, die 
rasch wachsenden grenzüberschreitenden privat- und schattenwirtschaftlichen Aktivitäten - vom Afrikahandel über Produktpiraterie und Großschmuggel bis hin zur Internetkriminalität - im Zaum zu halten. Chinas Image wird nicht nur durch Großmachtpolitik, sondern auch durch vielfältige irreguläre Geschäftspraktiken auf vielen Märkten zunehmend belastet. China hat von einem offenen, liberalen Welthandelssystem in besonderem Maße profitiert. Nun läuft es Gefahr, als selbstsüchtiger Globalisierungsgewinner wahrgenommen zu werden, der sich zentralen Regeln der globalen Wirtschaft entzieht und deshalb zur Zielscheibe protektionistischer Maßnahmen und in kostspielige Handelskriege verwickelt werden kann.

Zusammenfassend lässt sich festhalten, dass es in der Tat viele Faktoren und Risiken gibt, die einen anhaltenden wirtschaftlichen Aufstieg Chinas verhindern können. Allerdings sind die virulentesten Krisenfaktoren häufig in anderen Entwicklungen und Indikatoren zu finden, als es westliche Standardmodelle nahelegen. Die besondere Kombination von langfristiger Prioritätensetzung und dezentralen Politikexperimenten hat der chinesischen Wirtschaftspolitik eine unerwartete Anpassungsund Leistungsfähigkeit ermöglicht. In dieser Hinsicht könnte China für das volatile Umfeld des 21. Jahrhunderts besser gerüstet sein als viele der wirtschaftlich und politisch angeschlagenen, institutionell bislang wenig anpassungsfähigen OECDÖkonomien. ${ }^{4}$

\section{Literatur}

Heilmann, Sebastian, 2010: Economic governance: Authoritarian upgrading and innovative potential, in: Joseph Fewsmith (Hrsg.), China today, China tomorrow, Lanham, 109-126.

Heilmann, Sebastian/Shih, Lea, 2013: The Rise of Industrial Policy in China, 1978-2012. Harvard-Yenching Institute Working Paper Series.

Lardy, Nicholas R., 2012: Sustaining China's economic growth after the gobal financial crisis, Washington.

Lardy, Nicholas R./Borst, Nicholas, 2013: A blueprint for rebalancing the Chinese economy, Peterson Institute for International Economics Policy Brief Number PB13-02, February 2013.

Pettis, Michael, 2013: The great rebalancing. Trade, conflict, and the perilous road ahead for the world economy, Princeton/Oxford.

4 Vergleiche Heilmann 2010. 
Schmidt, Dirk/Heilmann, Sebastian, 2012: Außenpolitik und Außenwirtschaft der Volksrepublik China, Wiesbaden.

Taleb, Nassim Nicholas, 2007: The Black Swan, London/New York.

Taleb, Nassim Nicholas, 2012: Antifragile, London/New York.

Korrespondenzanschrift:

Prof. Dr. Sebastian Heilmann

Fachbereich III Politikwissenschaft

Lehrstuhl für Vergleichende Regierungslehre / Politik und Wirtschaft Chinas

Universität Trier

D-54286 Trier

E-Mail: heilmann@uni-trier.de

Dr. Dirk Schmidt

Fachbereich III Politikwissenschaft

Lehrstuhl für Vergleichende Regierungslehre / Politik und Wirtschaft Chinas Universität Trier

D-54286 Trier

E-Mail: dirk.schmidt@uni-trier.de 